\title{
Multi-catheter interstitial brachytherapy for partial breast irradiation: an audit of implant quality based on dosimetric evaluation comparing intra-operative versus post-operative placement
}

\author{
Lavanya Gurram, MD!, Tabassum Wadasadawala, MD', Kishor Joshi, DipRP2, Reena Phurailatpam, DipRP2, \\ Siji Paul, DipRP², Rajiv Sarin, MD, FRCR' \\ 'Department of Radiation Oncology, ${ }^{2}$ Department of Medical Physics, Advanced Centre for Treatment, Research \& Education in Cancer. \\ Tata Memorial Centre, Navi Mumbai, India
}

\begin{abstract}
Purpose: The use of multicatheter interstitial brachytherapy (MIB) for accelerated partial breast irradiation (APBI) in early breast cancer (EBC) patients outside the trial setting has increased. Hence, there is a need to critically evaluate implant quality. Moreover, there is a scarcity of reports using an open cavity technique. We report the dosimetric indices of open and closed cavity MIB techniques.

Material and methods: The dosimetric parameters of 60 EBC patients treated with MIB (open and closed cavity) who underwent three dimensional, computerized tomography (CT) based planning for APBI from November 2011 to July 2015 were evaluated. Coverage Index (CI), Dose Homogeneity Index (DHI), Conformity Index (COIN), Plan Quality Index (PQI), and Dose Non-uniformity Index (DNR) were assessed.

Results: Forty-one patients underwent open cavity and 19 patients underwent closed cavity placement of brachytherapy catheters. The median number of planes was 4 and median number of needles was 20 . Median dose was $34 \mathrm{~Gy}$ with dose per fraction of $3.4 \mathrm{~Gy}$, given twice a day, 6 hours apart. The $\mathrm{D}_{90}$ of the cavity and clinical target volume (CTV) were $105 \%$ and $89 \%$, respectively. The median doses to the surgical clips were greater than $100 \%$. The median CI of the cavity and CTV was 0.96 and 0.82 , respectively. The DHI and COIN index of the CTV was 0.73 and 0.67 . There were no significant differences in the dosimetric parameters based on whether the technique was done open or closed.

Conclusions: Critical evaluation of the dosimetric parameters of MIB-APBI is important for optimal results. While the open and closed techniques have similar dosimetry, our institutional preference is for an open technique which eases the procedure due to direct visualization of the tumor cavity.
\end{abstract}

Key words: APBI, brachytherapy, breast cancer, dosimetry, implant quality.

\section{Purpose}

Multicatheter interstitial brachytherapy (MIB) in breast cancer is being practiced over the past four decades to deliver tumor bed boost after whole breast radiotherapy (WBRT) and in accelerated partial breast irradiation (APBI) [1]. Accelerated partial breast irradiation gained tremendous popularity following US FDA approval of MammoSite ${ }^{\circledR}$ in 2002, as it offered a technically simpler method of delivering radiation therapy [2]. This resulted in aggressive adoption of a new technology with increasing use of APBI outside of the clinical trial setting. However, MIB is the oldest and time tested technique of delivering breast brachytherapy with respect to mature clinical outcome data [3]. In fact, it is being practiced since almost a decade before the guidelines for APBI were formulated [4,5]. As it is technically demanding and delivered without the need of any expensive device, it is not a very popular technique. Computed tomography (CT) based planning for MIB is a recent concept that has provided the clinicians an excellent opportunity not only to accurately delineate the target volume but also enables better planning and visualization of three dimensional volumetric data [6,7]. It is advisable to evaluate the quality of implant with the standard dosimetric parameters as it has a critical impact on the long term outcomes as well as on acute and late sequelae. In addition to the adequate coverage of the target volume 
for acceptable local control rates, dose homogeneity, ipsilateral breast, and skin doses are critical to avoid long term poor cosmetic outcomes. The standard dosimetric indices serve as a surrogate to estimate the risk of developing local recurrence and breast fibrosis $[8,9]$. This study reports the quality of MIB for APBI performed in suitable early stage breast cancer patients at our institution, based on dosimetric evaluation. We compared the indices based on the timing and technique (intra-operative open cavity vs postoperative closed cavity technique). The technical details of the two approaches has been explained in the subsequent section.

\section{Material and methods}

The dosimetric details of 60 patients treated with APBI between November 2011 and July 2015 with high-dose-rate (HDR) MIB using 3DCT based planning were evaluated.

\section{Selection criteria}

1. Age $>45$ years; 2 . Tumor size of $\leq 3 \mathrm{~cm} ; 3$. Lymph node negative status; 4 . Margins $\geq 2 \mathrm{~mm} ; 5$. Unicentric and unifocal; 6 . Invasive ductal carcinoma with no extensive intra-ductal component; 7 . No neoadjuvant chemotherapy prior to surgery; 8 . No extensive lympho-vascular emboli.

\section{Implant technique}

Patients underwent brachytherapy either in intraoperative or postoperative setting depending on the time of referral to our institute. If the patient was referred per primum i.e. pre-operatively, the patient was taken up for breast conservation surgery with intra-operative implant with open cavity technique. Patients were assessed pre-operatively for suitability with respect to clinico-radiological factors. The tumor bed was visualized directly and surgical clips were placed to mark the extent of the tumor cavity. After surface marking of the target volume, the procedure was performed with the insertion of stainless steel rigid needles in multiple planes depending on the excision volume in a triangular geometry. The steel needles were replaced with flexible plastic catheters and held in place with the help of steel buttons and plastic beads secured with adhesive tape. Lumpectomy cavity was closed after insertion of the needles. In case of adverse features on frozen section or histopathology report, implant procedure was either abandoned or converted into boost after 4 fractions. A gap of 3 weeks was maintained between initiation of adjuvant chemotherapy after brachytherapy to avoid any wound healing complications.

In the setting of patients who had already undergone excision biopsy or breast conserving surgery, suitable patients were considered for postoperative implant, which was done as a closed cavity technique. A pre-planning CT scan was obtained in all the patients who underwent postoperative implant to identify the tumor bed in the form of surgical clips or post-operative changes and decide implant geometry. In such cases, implant was performed 3-6 weeks after surgery or 3-4 weeks after completion of adjuvant chemotherapy. In either case, the procedure was planned under general anesthesia with a free hand or template technique.
After the procedure, a non contrast CT scan of the thoracic region was acquired in all the patients in supine position with a slice thickness of 1-3 mm depending upon the implant orientation. Radio-opaque dummies were inserted into the catheters before the acquisition of scans. Care was taken to align the patients for better visualization of the planar geometry and for catheter reconstruction. In the intra-operative setting, CT scan was done after 48 hours to reduce the postoperative edema and allow seroma collection. In the post-operative setting, CT scan was taken on the same day of the procedure.

\section{Image segmentation}

The CT images were transferred to Oncentra planning system (Version 4.3; Nucletron, Elekta, Stockholm, Sweden), on which the target volume segmentation and planning was performed. The cavity was identified with the help of seroma and surgical clips in the intra-operative setting, contoured in all the slices and re-checked on the sagittal and coronal views of the reconstructed images. In the post-operative setting when seroma was not identified, all the post-operative changes were included in the tumor bed along with the surgical clips. A margin of 1-1.5 cm was uniformly expanded, which was edited manually to create a clinical target volume (CTV). The CTV was edited $5 \mathrm{~mm}$ anteriorly from the skin surface and posteriorly from the chest wall. Ipsilateral breast was contoured. The recently published recommendations from GEC ESTRO (Groupe Européen de Curiethérapie - European Society for Radiotherapy and Oncology) Breast Cancer Working Group on target definition and target delineation for accelerated or boost partial breast irradiation using multicatheter interstitial brachytherapy after breast conserving closed cavity surgery provide an excellent guide for future [10].

\section{Planning}

Three dimensional CT based planning was done in all patients after multi-planar reconstruction of catheters. Computed tomography images aided in accurate visualization of catheter positions, and optimal evaluation of dose received by CTV. The main aim was to encompass the CTV by the prescription dose without compromising the normal tissue sparing. Graphical and geometric optimizations were done to achieve better dose distributions if required. Each plan was evaluated slice by slice with respect to the coverage of target volumes and to avoid the ballooning of isodoses to avoid high dose regions in the normal breast and skin. Maximum skin dose was limited to less than $70 \%$ of the prescribed dose. Dose to the tumor bed (TB) clips was evaluated separately by placing points at the surgical clips for dose calculations. F-factor was kept uniform for all implants at 0.85 .

\section{Doses}

A dose of 34 Gy in 10 fractions was prescribed to the CTV, which was delivered over a period of 5-7 days with 2 fractions delivered daily with a gap of 6 hours in between the 2 fractions. 
Table 1. Definitions of dosimetric indices

\begin{tabular}{|c|c|c|c|}
\hline Parameter & Definition & Formula & Importance \\
\hline Coverage Index (CI) & $\begin{array}{l}\text { Fraction of the target volume receiving } \\
\text { a dose } \geq \text { reference dose }\end{array}$ & $\begin{array}{c}\mathrm{Cl}(\mathrm{CTV}): \mathrm{CTV}_{100 \%} / \mathrm{V}_{\mathrm{CTV}} \\
\mathrm{Cl}(\mathrm{TB}): \mathrm{TBV}_{100 \%} / \mathrm{V}_{\mathrm{TB}}\end{array}$ & $\begin{array}{l}\text { How much target covered } \\
\text { by } 100 \% \text { dose }\end{array}$ \\
\hline $\begin{array}{l}\text { Dose Homogeneity } \\
\text { Index (DHI) }\end{array}$ & $\begin{array}{l}\text { Target volume enclosed by the prescribed } \\
\text { dose and that enclosed by a volume, } \\
\text { in which the dose is } 50 \% \text { higher than } \\
\text { the prescribed dose }\end{array}$ & $\begin{array}{c}\text { DHI: } \\
\mathrm{V}_{100 \%}-\mathrm{V}_{150 \%} / \mathrm{V}_{100 \%}\end{array}$ & $\begin{array}{l}\text { Higher DHI - } \\
\text { uniform distribution of dose }\end{array}$ \\
\hline $\begin{array}{l}\text { Conformity Index } \\
\text { (COIN) }\end{array}$ & $\begin{array}{c}\text { Coverage of CTV by reference dose and also } \\
\text { unwanted irradiation of normal tissues } \\
\text { outside CTV }\end{array}$ & $\begin{array}{c}\mathrm{C} 1 \times \mathrm{C} 2 \\
\mathrm{C} 1: \mathrm{CTV}_{\text {ref }} / \mathrm{CTV} \\
\mathrm{C} 2: \mathrm{CTV}_{\text {ref }} / \mathrm{V}_{\text {ref }}\end{array}$ & $\begin{array}{c}\text { Spillage of dose outside } \\
\text { target }\end{array}$ \\
\hline Plan Quality Index (PQI) & Sum of $\mathrm{Cl}, \mathrm{DHI}, \mathrm{COIN}$ & PQI: $\mathrm{Cl}+\mathrm{DHI}+\mathrm{COIN}$ & Implant quality \\
\hline $\begin{array}{l}\text { Dose Non-homogeneity } \\
\text { Ratio (DNR) }\end{array}$ & $\begin{array}{l}\text { Ratio of high dose volume to low dose } \\
\text { (reference volume) }\end{array}$ & DNR: $V_{150 \%} / V_{\text {ref }}$ & $\begin{array}{l}\text { Lower DNR - } \\
\text { uniform dose distribution }\end{array}$ \\
\hline $\begin{array}{l}\text { Overdose Volume Index } \\
(\mathrm{OVI})\end{array}$ & $\begin{array}{l}\text { Fraction of CTV receiving a dose equal to or } \\
\text { greater than two times the reference dose }\end{array}$ & OVI: $\mathrm{CTV}_{200} / \mathrm{CTV}_{100}$ & $\begin{array}{l}\text { Lower OVI- } \\
\text { better homogeneity }\end{array}$ \\
\hline $\begin{array}{l}\text { External Volume Index } \\
(\mathrm{EVI})\end{array}$ & $\begin{array}{l}\text { Volume of normal tissue outside CTV receiv- } \\
\text { ing dose more than or equal to reference dose }\end{array}$ & EVI: $\left(V_{100} C T V_{100}\right) / V_{C T V}$ & $\begin{array}{l}\text { Lower EVI- } \\
\text { lesser normal tissue dose }\end{array}$ \\
\hline
\end{tabular}

$T B$-tumor bed, TBV - tumor bed volume, $V_{100}$ - percentage volume of the CTV receiving at least $100 \%$ of the prescribed dose, $V_{150}-$ percentage volume of the CTV receiving $150 \%$ of the prescribed dose, $D_{90}$ - minimum percentage dose received by $90 \%$ of the target volume, $D_{100}-$ minimum percentage dose received by $100 \%$ of the target volume

Table 2. Dose and volumetric comparison between the two techniques

\begin{tabular}{|c|c|c|}
\hline \multirow[t]{2}{*}{ Parameters } & \multicolumn{2}{|c|}{ Median (range) } \\
\hline & Intraoperative & Postoperative \\
\hline Number of catheters & $20(11-25)$ & $20(16-23)$ \\
\hline Number of planes & $4(3-5)$ & $4(2-4)$ \\
\hline Total dose (Gy) & $34(13.6-34)$ & $34(34)$ \\
\hline Dose per fraction (Gy) & 3.4 & 3.4 \\
\hline Volume of TB (cc) & $31.4(6.2-92.8)$ & $27(22-101.3)$ \\
\hline Volume of CTV (cc) & $103.8(49-193.7)$ & $102(42.1-200.7)$ \\
\hline Volume of breast (cc) & $\begin{array}{c}770 \\
(260.0-2381.0)\end{array}$ & $\begin{array}{c}972.3 \\
(552.7-1783.0)\end{array}$ \\
\hline CTV $150 \%$ (cc) & $23.4(11.5-49)$ & $22.1(7.7-48.3)$ \\
\hline CTV $200 \%$ (cc) & $7.5(1.8-12.5)$ & $7.8(1.2-17.2)$ \\
\hline $\mathrm{V}_{150 \%}(\mathrm{cc})$ & $28.3(13.6-52.1)$ & $29(14.6-59.2)$ \\
\hline$V_{200 \%}(\mathrm{cc})$ & $10.5(5.6-18.7)$ & $12(6.1-22.2)$ \\
\hline $\mathrm{D}_{90}$ cavity $(\%)$ & $104.8(62-128)$ & $106.8(68-133.2)$ \\
\hline $\mathrm{D}_{90} \mathrm{CTV}(\%)$ & $89(69-132.23)$ & $88(66-112.4)$ \\
\hline \multicolumn{3}{|l|}{ Surgical clips dose (\%) } \\
\hline 1 & 122 & 113 \\
\hline 2 & 126 & 115 \\
\hline 3 & 121 & 115 \\
\hline 4 & 129 & 113 \\
\hline
\end{tabular}

TB - tumor bed, CTV - clinical target volume, $V_{150 \%}$ - percentage volume of the $C T V$ receiving $150 \%$ of the prescribed dose, $V_{200 \%}$ - percentage volume of the CTV receiving $200 \%$ of the prescribed dose, $D_{90}$-minimum percentage dose received by $90 \%$ of the target volume.

\section{Definitions and dosimetric indices}

The definitions and importance of dosimetric indices used have been explained in Table 1.

\section{Statistical methods}

The indices were calculated using the formulae in Microsoft Excel 2007 as shown in Table 1. The clinical data along with the dosimetric data was entered in SPSS version 20 (SPSS Inc., Chicago, IL, USA). The medians of the various volumes were calculated. Comparison of the indices between the two techniques was done using two sample $t$-test. A $p$ value of less than 0.05 was considered significant.

\section{Results}

\section{Implant details}

Of the 60 patients, 41 underwent intra-operative implant and 19 in the postoperative setting. The median age at presentation was 60 years (range: $45-73$ years) with a median tumor size of $2.2 \mathrm{~cm}$ (range: 1.0-3.0 cm). The most common histology was infiltrating ductal carcinoma (IDC), grade III $(n=44)$, followed by grade II $(n=14)$. Grade I IDC was seen in two patients. Ductal carcinoma in situ (DCIS) was present in 28 patients. Estrogen receptor positivity was seen in 21 patients, Her-2 positivity was seen in 10 patients, and 10 patients were triple receptor negative. Implant was not performed in women with pure DCIS.

The median number of planes was 4 and median number of tubes was 20. The treatment of one patient who underwent intra-operative placement of catheters was terminated after 4 fractions and dose of 13.6 Gy was delivered in view of adverse risk feature (lymph node positive) in the final histopathology report. The patient subsequently received whole breast radiotherapy and the 
Table 3. Comparison of dosimetric indices between the two techniques

\begin{tabular}{lccc} 
Parameters & $\begin{array}{c}\text { Intraoperative } \\
\text { (open cavity) }\end{array}$ & $\begin{array}{c}\text { Postoperative } \\
\text { (closed cavity) }\end{array}$ & $\begin{array}{c}\text { Entire cohort } \\
\text { Median (range) }\end{array}$ \\
\hline Cl cavity & 0.92 & 0.91 & $0.96(0.56-1)$ \\
\hline CI CTV & 0.83 & 0.80 & $0.82(0.65-1)$ \\
\hline DHI & 0.72 & 0.72 & $0.73(0.42-0.84)$ \\
\hline COIN & 0.68 & 0.65 & $0.67(0.59-0.87)$ \\
\hline PQI & 2.24 & 2.18 & $2.24(1.78-2.53)$ \\
\hline OVI & 0.08 & 0.08 & $0.08(0.02-0.17)$ \\
\hline EVI & 0.18 & 0.33 & $0.16(0.00-0.60)$ \\
\hline DNR & 0.31 & 0.25 & $0.28(0.14-0.60)$
\end{tabular}

p value - not significant, CI - Coverage Index, DHI - Dose Homogeneity Index, COIN - Conformity Index, PQI - Plan Quality Index, OVI - Overdose Volume Index, EVI - External Volume Index, DNR - Dose Non-homogeneity Ratio

dose delivered by MIB was converted into boost. In addition to volumetric dose evaluation, point dose estimation of the surgical clips defining the cavity was also done. Tables 2 and 3 compare the dosimetric characteristics of patients treated with the two techniques.

\section{Discussion}

In our present report, we have critically evaluated the implant quality at our institute using various dosimetric parameters as per the existing guidelines and the adherence to the same is shown in Table 4. At our center, we have replaced 2D orthogonal X-ray based planning with 3D planning due to the inherent advantages of CT scan, in the form of accurate delineation of the structures and optimal evaluation of dose distributions within and outside the target volume [7]. The advantages of 3D planning have been reported in a number of studies. Major et al., evaluated the dose volume parameters of implants with post implant CT scans that were initially planned with orthogonal $X$ rays. It was seen that the target coverage, DHI, and COIN significantly improved with three dimensional planning [11]. Borger et al. reported an increase in the rate of breast fibrosis with an increase in implant volumes in patients treated with low-dose-rate MIB using conventional 2D planning [12].

With the increase in implementation of dose point graphical optimization, there is a risk of increasing the high dose regions, which has a bearing on the late sequelae such as fibrosis and if not evaluated properly, can lead to necrosis of the soft tissue. Moreover, while doing this one has to be cautious with respect to any change in the implant normalization and the resultant dose delivered. In the current study, graphical optimization was primarily used to reduce the skin dose taking care of plan normalization value, which was uniformly kept at 0.85 for all cases. In the retrospective dosimetric analysis by Kestin et $a l$. it was observed that the high dose regions within the treatment volumes increased dramatically from $26 \mathrm{cc}$ to $70 \mathrm{cc}$ when the dwell times were increased to improve coverage [13]. The volume receiving $150 \%$ of the prescribed dose in our series was $23 \mathrm{cc}$, which is much low- er as compared to the acceptable limit recommended by the RTOG. Ott et al. recommended CTV volumes of up to $180 \mathrm{cc}$ for optimal outcomes [14]. In our study, the median CTV volume was $103 \mathrm{cc}$ with a small number of patients exceeding $200 \mathrm{cc}$.

Cuttino et al. reported the improvement in coverage of CTV of up to $95 \%$ and DHI of 0.82 over years as the technique was adopted and established at their institute [15]. In the updated report of their experience, Cholewka et al. reported $95 \%$ coverage of CTV, DHI of 0.68 , and COIN of 0.75 [16]. We adopted the evaluation of PQI of implants similar to the above authors to qualitatively estimate our procedure [16]. On the contrary, when we analyzed our results over the years, it was seen that all the dosimetric indices except tumor bed coverage improved in the later couple of years. Major et al. reported a DNR of 0.33 and COIN of 0.68 in patients who underwent CT based planning $[17,18]$. Our dosimetric outcome is similar to that reported in literature, which is summarized in Table 5. The main difference is the average volume and coverage of the CTV. This is mainly because in our institute, the common practice is intra-operative open cavity procedure (post lumpectomy), which results in a larger CTV volume mainly due to the accumulation of air and

Table 4. Adherence of implant quality to standard guidelines

\begin{tabular}{lccc} 
Parameter & RTOG/ABS [8] & $\begin{array}{c}\text { Present } \\
\text { Study }\end{array}$ & GEC-ESTRO [9] \\
\hline $\mathrm{DHI}$ & $\geq 0.75$ & 0.73 & - \\
\hline $\mathrm{V}_{150}$ & $\leq 70 \mathrm{cc}$ & $23.0 \mathrm{cc}$ & - \\
\hline $\mathrm{V}_{200}$ & $\leq 20 \mathrm{cc}$ & $7.5 c c$ & - \\
\hline DNR & - & 0.28 & $<0.35$ \\
\hline Size of CTV & - & $16-200 c c$ & $40-150 c c$
\end{tabular}

RTOG/ABS - Radiation Therapy Oncology Group/American Brachytherapy Society, GEC-ESTRO - Groupe Européen de Curiethérapie - European Society for Radiotherapy and Oncology, CTV - clinical target volume, DHI - Dose Homogeneity Index, DNR - Dose Non-homogeneity Ratio, $V_{150 \%}$ - percentage volume of the CTV receiving $150 \%$ of the prescribed dose, $V_{200 \%}$ - percentage volume of the CTV receiving $200 \%$ of the prescribed dose 
Table 5. Comparison of the dosimetric parameters amongst various studies

\begin{tabular}{lcccc}
\hline Parameters & Major et al. [6] & Dutta et al. [19] & Cholewka et al. [16] & Present Study \\
\hline Number of patients & 49 & 15 & 191 & 60 \\
\hline Number of catheters & $15(6-28)$ & $10(9-16)$ & $14(7-18)$ & $20(15-24)$ \\
\hline Number of planes & $4(3-6)$ & $2(2-3)$ & $3(2-5)$ & $4(3-5)$ \\
\hline Volume CTV (cc) & $66.4(15.5-176)$ & $107.5(56.8-196.9)$ & $71.6(17.4-226.5)$ & $103.3(42.1-258.8)$ \\
\hline Cl (CTV) & NR & $0.93(0.63-0.99)$ & NR & $0.82(0.65-1)$ \\
\hline COIN & $0.68(0.51-0.82)$ & NR & $0.69(0.37-0.84)$ & $0.67(0.59-0.87)$ \\
\hline DHI & $0.65(0.50-0.76)$ & $0.65(0.452-0.79)$ & $0.66(0.40-0.78)$ & $0.73(0.42-0.84)$ \\
\hline PQI & NR & $1.35(1.19-1.65)$ & NR & $2.24(1.78-2.53)$
\end{tabular}

CTV-clinical target volume, Cl-Coverage Index, COIN - Conformity Index, DHI-Dose Homogeneity Index, PQI-Plan Quality Index

seroma in the tumor bed. The lower coverage of the CTV is attributed to the editing of the CTV in the air region of the seroma close to the skin. Higher median number of tubes and planes is also related to the larger CTV volume. It has been observed in our study that open cavity MIB is equally efficacious as that of closed cavity technique with respect to $\mathrm{CI}$ and DHI. Considering the advantage of direct visualization of the tumor bed and excellent multi-disciplinary co-ordination, intra-operative procedures are preferred at our institute.

\section{Conclusions}

Critical evaluation of MIB implants for APBI and compliance of dosimetric parameters with the existing recommendations is very important to predict outcomes. This ensures quality assurance of the procedure and improved learning curve for optimal results. Our study reports dosimetric outcome comparable to that of the RTOG (Radiation Therapy Oncology Group) and GEC-ESTRO guidelines and better than few of the reported series. In our report, no difference in implant quality was seen between intra-operative placement of catheters and post-operative implants. We encourage intra-operative technique in properly selected patients in view of added advantage of direct visualization of the tumor cavity.

\section{Disclosure}

Authors report no conflict of interest.

\section{References}

1. King T, Bolton J, Kuske R et al. Long-term results of wide-field brachytherapy as the sole method of radiation therapy after segmental mastectomy for Tis,1,2 breast cancer. Am J Surg 2000; 180: 299-304.

2. Skowronek J, Wawrzyniak-Hojczyk M, Ambrochowicz K. Brachytherapy in accelerated partial breast irradiation (APBI) - review of treatment methods. J Contemp Brachytherapy 2012; 4: 152-164.

3. Strnad V, Ott OJ, Hildebrandt G et al. 5-year results of accelerated partial breast irradiation using sole interstitial multicatheter brachytherapy versus whole-breast irradiation with boost after breast-conserving surgery for low-risk invasive and in-situ carcinoma of the female breast: a randomized, phase 3, non-inferiority trial. Lancet 2016; 387: 229-238.

4. Polgár C, Van Limbergen E, Pötter R et al. Patient selection for accelerated partial-breast irradiation (APBI) after breast-conserving surgery: recommendations of the Groupe Européen de Curiethérapie-European Society for Therapeutic Radiology and Oncology (GEC-ESTRO) breast cancer working group based on clinical evidence (2009). Radiother Oncol 2010; 94: 264-273.

5. Tang JI, Tan PW, Koh VY et al. Multi-catheter interstitial accelerated partial breast irradiation - tips and tricks for a good insertion. J Contemp Brachytherapy 2014; 6: 85-90.

6. Major T, Polgár C, Lövey K et al. Dosimetric characteristics of accelerated partial breast irradiation with CT image-based multicatheter interstitial brachytherapy: A single institution's experience. Brachytherapy 2011; 10: 421-426.

7. Sharma S, Budrukkar A, Upreti R et al. Dosimetric comparison of conventional radiograph- and three-dimensional computed tomography-based planning using dose volume indices for partial breast intraoperative implants. Clin Oncol 2008; 20: 46-52

8. NSABP protocol B-39/RTOG protocol 0413. A randomized phase III study of conventional whole breast irradiation (WBI) versus partial breast irradiation (PBI) for women with stage 0, I, II breast cancer. http://www.rtog.org/members/ protocols/0413/0413.pdf

9. European Brachytherapy Breast Cancer GEC-ESTRO working group. Phase III multicenter trial. Interstitial brachytherapy alone versus external beam radiation therapy after breast conserving surgery for low risk invasive carcinoma and low risk duct carcinoma in-situ (DCIS) of the female breast. http://www.apbi.uni-erlangen.de/index.html

10. Strnad V, Hannoun-Levi JM, Guinot JL et al. Recommendations from GEC ESTRO Breast Cancer Working Group (I): Target definition and target delineation for accelerated or boost Partial Breast Irradiation using multicatheter interstitial brachytherapy after breast conserving closed cavity surgery. Radiother Oncol 2015; 115: 342-348.

11. Major T, Fodor J, Takácsi-Nagy Z et al. Evaluation of HDR interstitial breast implants planned by conventional and optimized CT-based dosimetry systems with respect to dose homogeneity and conformality. Strahlenther Onkol 2005; 181: 89-96.

12. Borger J, Kemperman H, Smitt HS et al. Dose and volume effects on fibrosis after breast conservation therapy. Int J Radiat Oncol Biol Phys 1994; 30: 1073-1081.

13. Kestin L, Jaffray D, Edmundson G et al. Improving the dosimetric coverage of interstitial high-dose-rate breast implants. Int J Radiat Oncol Biol Phys 2000; 46: 35-43. 
14. Ott O, Hildebrandt G, Pötter R et al. Accelerated partial breast irradiation with multi-catheter brachytherapy: Local control, side effects and cosmetic outcome for 274 patients. Results of the German-Austrian multi-centre trial. Radiother Oncol 2007; 82: 281-286.

15. Cuttino L, Todor D, Arthur D. CT-guided multi-catheter insertion technique for partial breast brachytherapy: Reliable target coverage and dose homogeneity. Brachytherapy 2005; 4: 10-17.

16. Cholewka A, Szlag M, Białas B et al. The importance of the implant quality in APBI - Gliwice experience. Dosimetric evaluation. J Contemp Brachytherapy 2013; 4: 227-231.

17. Major T, Fröhlich G, Lövey K et al. Dosimetric experience with accelerated partial breast irradiation using image-guided interstitial brachytherapy. Radiother Oncol 2009; 90: 48-55.

18. Major T, Fröhlich G, Polgar C. Assessment of dose homogeneity in conformal interstitial breast brachytherapy with special respect to ICRU recommendations. J Contemp Brachytherapy 2011; 3: 150-155.

19. Dutta S, Sharma S, Singh R et al. Quality assessment of interstitial implants in high-dose-rate brachytherapy after lumpectomy in patients of early stage breast cancer. Indian J Surg Oncol 2010; 1: 294-302 